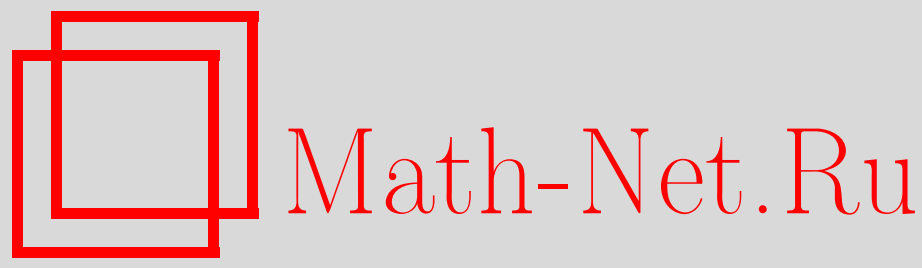

Ю. Хонконен, Модели реакций в стохастической теории поля, ТМФ, 2011, том 169, номер 1, 112-123

DOI: https://doi.org/10.4213/tmf6713

Использование Общероссийского математического портала Math-Net.Ru подразумевает, что вы прочитали и согласны с пользовательским соглашением http://www . mathnet.ru/rus/agreement

Параметры загрузки:

IP: 54.172 .240 .79

26 апреля 2023 г., 10:31:14

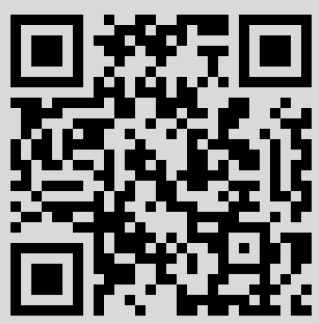




\title{
Ю. Хонконен* МОДЕЛИ РЕАКЦИЙ В СТОХАСТИЧЕСКОЙ ТЕОРИИ ПОЛЯ
}

\begin{abstract}
Обсуждаются и сравниваются два популярных метода, применяемых при анализе флуктуаций числа активных агентов: подход Мартина-Сиггиа-Розе, обычно связываемый с описанием кинематики флуктуаций с помощью уравнения Ланжевена, и подход Дои, непосредственно связанный с описанием стохастических процессов рождения и уничтожения в терминах управляющего уравнения.
\end{abstract}

Ключевые слова: ренормализационная группа, кинетика реакций, случайные источники и стоки, размерный анализ, эффективное действие.

\section{1. ВВЕДЕНИЕ}

Флуктуации числа активных агентов, участвующих в химических реакциях, в динамике популяций и в моделях военных боестолкновений, приводят к существенным последствиям для макроскопической динамики системы. Уже в течение более чем трех десятилетий для анализа этих флуктуаций применяются мощные методы квантовой теории поля.

Учет флуктуаций в физических процессах часто реализуется с помощью стохастических дифференциальных уравнений (СДУ). Самый известный пример таких уравнений - это знаменитое уравнение Ланжевена, в котором случайное поле выступает в роли случайных источников и стоков некоторой динамической величины, и предполагается, что это уравнение способно описывать эффекты, привносимые микроскопическими степенями свободы.

C тех пор как было построено представление в виде функционального интеграла [1] для подхода Мартина-Сиггиа-Розе к решению стохастических задач [2], постоянно происходит обсуждение неоднозначностей, возникающих в указанной формулировке [3]-[5]. Неоднозначности возникают в силу двух обстоятельств. Во-первых, замена переменных, используемая в конструкции, приводит к появлению функционального определителя Якоби, который недоопределен. Во-вторых, в силу математической несогласованности уравнения Ланжевена с белым шумом (см., например, [6]) имеют место различные согласованные определения СДУ, влияющие также и на вид решения в форме функционального интеграла. Задача настоящей работы

\footnotetext{
*National Defence University, Helsinki, Finland. E-mail: juha.honkonen@helsinki.fi
} 
состоит в том, чтобы сформулировать в унифицированном виде следствия, вытекающие из обоих источников неоднозначности.

Множество стохастических задач в физике, химии, биологии и т. д. формулируются в терминах управляющего уравнения. В этом подходе вероятностное определение случайного процесса производится с помощью его словесного определения. Этот подход можно рассматривать как более фундаментальный по сравнению с подходом, основанным на применении уравнения Ланжевена [7].

Решения системы управляющих уравнений для стохастического процесса можно также выразить в терминах функционального интеграла с помощью “вторичного квантования" классических переменных [8], [9]. Описания стохастической задачи, задаваемые двумя указанными подходами, часто приводят к различным стохастическим теориям поля. Происхождение этих несогласований, равно как и соотношения между соответствующими теориями поля, обсуждаются в настоящей работе на основе реакции коагуляции $A+A \rightarrow A$.

В большинстве моделей стохастических процессов степень убывания переменной типа плотности стремится к нулю при стремлении к нулю само́й плотности. Тем не менее встречаются модели, в которых степень убывания не зависит от плотности. В этом случае динамическое действие стохастической теории поля становится гораздо более сложным, что также сильно усложняет асимптотический анализ. Примером такой модели служит реакция, ограниченная диффузией (РОД), которая основана на модели боестолкновения Ланчестера с применением современной боевой техники, обсуждаемая в настоящей работе. Также встречаются задачи двух представителей, в которых определение поведения масштабирующих функций в пределе больших времен оказывается чрезвычайно сложной задачей, до сих пор не имеющей решения.

Работа построена следующим образом. В разделе 2 анализируются неоднозначности в определении функционального интеграла при решении СДУ. Обзор функционально-интегрального представления для одношаговых процессов приведен в разделе 3, где также исследуются эффекты, привносимые случайными стоками и источниками. В разделе 4 представлен асимптотический анализ с применением ренормализационной группы для РОД с двум представителями для стохастической модели боестолкновения Ланчестера. Заключение и обсуждение результатов содержатся в разделе 5 .

\section{2. ДИНАМИЧЕСКАЯ ТЕОРИЯ ПОЛЯ ДЛЯ СДУ}

Рассмотрим уравнение Ланжевена

$$
\frac{\partial \varphi}{\partial t}=V(\varphi)+f B(\varphi):=-K \varphi+U(\varphi)+f B(\varphi)
$$

где $f$ - гауссово случайное поле с нулевым средним, и корреляционную функцию

$$
\left\langle f(t, \mathbf{x}) f\left(t^{\prime}, \mathbf{x}^{\prime}\right)\right\rangle=\bar{D}\left(x-x^{\prime}\right)=\delta\left(t-t^{\prime}\right) D\left(\mathbf{x}-\mathbf{x}^{\prime}\right),
$$

где использована сокращенная форма записи $x=(t, \mathbf{x})$.

В качестве основного примера выберем модель А критической динамики [10], задаваемую уравнением Ланжевена

$$
\frac{\partial \varphi}{\partial t}=-\Gamma\left(-\nabla^{2} \varphi+a \varphi+\frac{\lambda}{6} \varphi^{3}\right)+f
$$


с кинетическим коэффициентом $\Gamma$ и корреляционной функцией $D\left(\mathbf{x}-\mathbf{x}^{\prime}\right)=2 \Gamma \delta(\mathbf{x}-$ $\left.\mathbf{x}^{\prime}\right)$. Здесь и ниже подразумевается, что осуществляются все необходимые интегрирования и суммирования.

Уравнение Ланжевена с белым шумом $f$ относительно времени оказывается математически несамосогласованным, поскольку интеграл по времени для шума $\int f d t$ представляет собой винеровский процесс, и поэтому производная по времени $\partial_{t} \varphi$ не существует ни при каком значении временно́го аргумента. СДУ представляет собой интегральное уравнение

$$
\varphi(t)=\varphi(0)+\int_{0}^{t}[-K \varphi+U(\varphi)] d \tau+\int_{0}^{t} B(\varphi) d W(t) .
$$

Чтобы интерпретировать уравнение (4) согласованным образом, необходимо определить интегрирование по винеровскому процессу $W$. Наиболее часто эта интерпретация использует стохастические интегралы Ито или Стратоновича. После того как определен стохастический интеграл, математическое ожидание и старшие моменты решения $\varphi$ СДУ (4) могут быть построены итерациями, на каждом шаге которых стохастические интегралы выражаются в терминах обычных интегралов (см., например, [6]).

Тем не менее начнем с набора корреляционных функций, состоящих из $\delta$-последовательностей по времени, т. е.

$$
\left\langle f(t, \mathbf{x}) f\left(t^{\prime}, \mathbf{x}^{\prime}\right)\right\rangle=\bar{D}\left(t, \mathbf{x} ; t^{\prime}, \mathbf{x}^{\prime}\right) \underset{t^{\prime} \rightarrow t}{\longrightarrow} \delta\left(t-t^{\prime}\right) D\left(\mathbf{x}, \mathbf{x}^{\prime}\right) .
$$

Из этого рассмотрения следует решение СДУ (1) в смысле Стратоновича [6].

Чтобы представить решение уравнения Ланжевена (1) в виде функционального интеграла, вычислим производящую функцию как среднее от линейной экспоненциальной функции решения $\varphi[f]$ уравнения Ланжевена над распределением вероятности случайного источника $f$ [1]. Если в дальнейшем представить эту экспоненциальную функцию в виде интеграла по вспомогательному полю, произвести замену переменных, чтобы явно ввести уравнение Ланжевена в данное представление, и использовать представление Фурье для $\delta$-функции, то в итоге получим

$$
\begin{aligned}
G(J) & =\left\langle e^{\varphi[f] J}\right\rangle=\int \mathcal{D} \varphi\langle\delta(\varphi-\varphi[f])\rangle e^{\varphi J}= \\
& =\int \mathcal{D} \varphi\left\langle\delta\left(-\partial_{t} \varphi+V(\varphi)+f B(\varphi)\right)|\operatorname{det} M|\right\rangle e^{\varphi J}= \\
& =\int \mathcal{D} \varphi \int \mathcal{D} \tilde{\varphi}\left\langle|\operatorname{det} M| e^{\tilde{\varphi}\left(-\partial_{t} \varphi+V(\varphi)+f B(\varphi)\right)}\right\rangle e^{\varphi J}
\end{aligned}
$$

Эта процедура приводит к возникновению действия Де Доминикиса-Янссена

$$
S[\varphi, \tilde{\varphi}, f]=\ln P[f]+\ln |\operatorname{det} M|+\tilde{\varphi}\left(-\partial_{t} \varphi+V(\varphi)+f B(\varphi)\right),
$$

где $P[f]$ - функционал плотности вероятности для поля источника $f$. В случае мультипликативного шума величина $\ln |\operatorname{det} M|$ зависит от поля $f$. Заметим, что в противоположность утверждениям, содержащимся в литературе [3], появление этого функционального определителя не связано со стохастическим характером уравнения Ланжевена (1): этот определитель возникает также и в представлении решения детерминистского уравнения (с заданным неслучайным полем $f$ ). 
Применяя петлевое разложение для множителя $\operatorname{det} M=e^{\operatorname{Tr} \ln M}$ в $(6)$, получим

$$
\operatorname{det} M=\operatorname{det}\left(\partial_{t}+K-U^{\prime}-f B^{\prime}\right)=\operatorname{det}\left(\partial_{t}+K\right) e^{-\Delta(0)\left(U^{\prime}+f B^{\prime}\right)},
$$

где использовано обозначение

$$
\begin{aligned}
\Delta(0)\left(U^{\prime}+f B^{\prime}\right)=\int & d t \int d \mathbf{x} \int d \mathbf{x}^{\prime} \Delta\left(t, \mathbf{x} ; t, \mathbf{x}^{\prime}\right) \times \\
& \times \int d u\left[\frac{\delta U\left(\mathbf{x}^{\prime}, \varphi(u)\right)}{\delta \varphi(t, \mathbf{x})}+f\left(t, \mathbf{x}^{\prime}\right) \frac{\delta B\left(\mathbf{x}^{\prime}, \varphi(u)\right)}{\delta \varphi(t, \mathbf{x})}\right] .
\end{aligned}
$$

Значение пропагатора (т. е. функции отклика поля $\varphi$ ) при совпадающих временнь́х аргументах, $\Delta(0):=\Delta\left(t, \mathbf{x} ; t, \mathbf{x}^{\prime}\right)$, остается параметром модели. Вводя обычную гауссову весовую функцию для $f$, получим динамическое действие

$$
S[\varphi, \tilde{\varphi}, f]=-\Delta(0)\left(U^{\prime}+f B^{\prime}\right)-\frac{1}{2} f(\bar{D})^{-1} f+\tilde{\varphi}\left[-\partial_{t} \varphi-K \varphi+U(\varphi)+f B\right] .
$$

Двумя популярными вариантами выбора величины этого параметра оказываются $\Delta(0)=0$ [5] и $\Delta(0)=\delta\left(\mathbf{x}-\mathbf{x}^{\prime}\right) / 2$ [4]. Это выглядит как выбор между интерпретациями СДУ Ито и Стратоновича, но следует иметь в виду, что случайность пока что не играет никакой роли.

Из функционального определителя Якоби получаются графы, сокращающие лишние диаграммы, возникающие в графическом представлении функционального интеграла в соответствии со стандартными правилами Фейнмана для динамического действия (10). Полезно найти итеративное решение простого СДУ

$$
\frac{\partial \varphi}{\partial t}=-K \varphi+f \varphi
$$

где $K$ представляет собой оператор, зависящий от времени и действующий на поле $\varphi$ (например, $K=-\nabla^{2}+a, a>0$ ), а $f$ - фиксированное поле. Соответствующее динамическое действие имеет вид

$$
S=-\Delta(0) f+\tilde{\varphi}\left(-\partial_{t} \varphi-K \varphi+f \varphi\right)
$$

и оно приводит к теории возмущений, в которой решение для поля $\varphi$ имеет вид

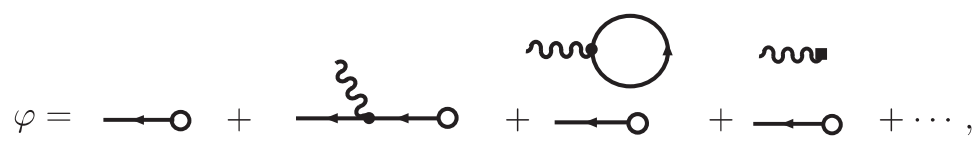

где кружком обозначены начальные условия $\chi$ однородного уравнения, волнистая линия отвечает случайному полю $f$, а жирная точка отвечает множителю при вершине, получающемуся из последнего члена уравнения Ланжевена (11). Черным квадратом в формуле (13) обозначен коэффициент $-\Delta(0)$, который представляет собой вершинный множитель, обусловленный определителем Якоби $-\Delta(0) f$ в динамическом действии.

При подходящем выборе $\Delta(0)$ суммы вкладов от графов с замкнутой петлей пропагатора и от графов, задаваемых определителем в правой части уравнения (13), 
взаимно сокращаются; при этом восстанавливается решение в виде древесных графов (11) с фиксированным $f$

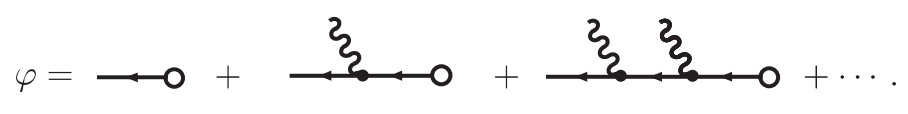

Поэтому в любом случае итеративное решение уравнения Ланжевена оказывается единственным (не зависящим от величины $\Delta(0)$ ), а потому оказывается единственным и пертурбативное решение, задаваемое функциональным интегралом.

Вычислив среднее от ряда (14) относительно плотности вероятности величины $f$, получим решение СДУ (11) для поля $\varphi$. Моменты распределения для величины $\varphi$ можно получить, если вычислить средние произведений рядов (14).

В случае распределения Гаусса с нулевым средним и с корреляционной функцией (2) динамическое действие, получаемое из СДУ (11), принимает вид

$$
S[\varphi, \tilde{\varphi}, f]=-\Delta(0) f-\frac{1}{2} f D^{-1} f+\tilde{\varphi}\left(-\partial_{t} \varphi-K \varphi+f \varphi\right) .
$$

Пертурбативное решение СДУ (11) задается теоремой Вика, применение которой графически сводится к замене каждой пары вставок $f$ корреляционной функцией $\bar{D}$, представленной неориентированной линией, с суммированием по всем возможным случаям. Например, графическое представление (14) приводит к представлению

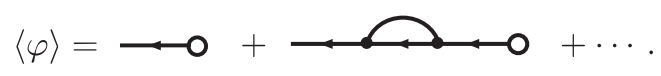

В пределе корреляций, имеющих характер белого шума по времени, использование запаздывающего пропагатора $\Delta$ приводит к значительному укорачиванию усредненного ряда по итерациям для $\varphi^{n}$, поскольку в этом пропагаторе присутствуют временны́е $\delta$-функции, участвующие в свертках концов цепей, составленных из запаздывающих пропагаторов. Только члены, в которых корреляционная функция умножается на единственный запаздывающий пропагатор, при этом не обращаются в нуль автоматически.

Например, однопетлевой граф в формуле (16) в случае белого по времени шума приводит к неопределенности, которая непосредственно связана с неопределенностью при интерпретации СДУ (11). При подстановке корреляционной функции белого шума в этот граф получим выражение

$$
\begin{array}{r}
\longrightarrow \sim \mathrm{O}^{\infty}=\int t_{1} \int d \mathbf{x}_{1} \int d \mathbf{x}_{2} \int d \mathbf{x}_{3} \Delta\left(t-t_{1}, \mathbf{x}-\mathbf{x}_{1}\right) \times \\
\times \Delta\left(0, \mathbf{x}_{1}-\mathbf{x}_{2}\right) D\left(\mathbf{x}_{1}, \mathbf{x}_{2}\right) \Delta\left(t_{1}, \mathbf{x}_{2}-\mathbf{x}_{3}\right) \chi\left(\mathbf{x}_{3}\right),
\end{array}
$$

в котором значение пропагатора при совпадающих временнь́х аргументах $\Delta\left(0, \mathbf{x}_{1}-\right.$ $\left.\mathbf{x}_{2}\right)=\theta(0) \delta\left(\mathbf{x}_{1}-\mathbf{x}_{2}\right)$ снова оказывается произвольным.

Если применить $\delta$-последовательность корреляционных функций (5), эту неопределенность можно явно разрешить, что приводит к выражению

$$
\frac{1}{2} \int d t_{1} \int d \mathbf{x}_{1} \int d \mathbf{x}_{3} \Delta\left(t-t_{1}, \mathbf{x}-\mathbf{x}_{1}\right) D\left(\mathbf{x}_{1}, \mathbf{x}_{1}\right) \Delta\left(t_{1}, \mathbf{x}_{1}-\mathbf{x}_{3}\right) \chi\left(\mathbf{x}_{3}\right)
$$


с множителем $1 / 2$, стоящим перед пространственной $\delta$-функцией. Эта процедура отвечает интерпретации СДУ (11) в смысле Стратоновича. Этот же результат можно формально получить, подправив определение пропагатора в соответствии с правилом $\Delta\left(0, \mathbf{x}-\mathbf{x}^{\prime}\right)=\delta\left(\mathbf{x}-\mathbf{x}^{\prime}\right) / 2$.

Можно получить более удобное описание, если принять соглашение, в соответствии с которым $\Delta(0)=0$. В этом случае детерминистскую часть динамического действия (15) необходимо подправить, чтобы из нее вытекал правильный ненулевой ответ для графов вида (17). Этого можно достичь с помощью добавления к действию члена

$$
\Delta S=\frac{1}{2} \tilde{\varphi} D(0) \varphi:=\frac{1}{2} \int d t \int d \mathbf{x} \tilde{\varphi}(t, \mathbf{x}) D(\mathbf{x}, \mathbf{x}) \varphi(t, \mathbf{x}) .
$$

Графически эта процедура сводится к замене однопетлевого графа с шумовой корреляционной функцией на новую вершину с коэффициентом $D(0) / 2$ :

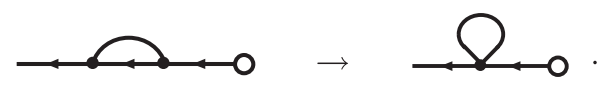

Можно непосредственно увидеть, что поправка (19) к динамическому действию отвечает регуляризации СДУ (11), при которой уравнение Стратоновича заменяется на уравнение Ито с тем же решением [6]. Это графическое рассуждение можно непосредственно обобщить на произвольный множитель при $f$, полиномиальный относительно $\varphi$.

Вернемся на время к итеративному решению уравнения (4), которое можно графически записать в виде древесного графа. Выбор стохастического интеграла показывает, когда вычисляются математические ожидания функций от $\varphi$. Комбинаторные правила винеровского процесса задаются теоремой Вика. Результаты вычислений математических ожиданий временнь́х интегралов от попарных произведений винеровских процессов различны для стохастических интегралов Ито и Стратоновича.

Практическое правило вычисления можно сформулировать следующим образом [6]: перепишем инкремент винеровского процесса в виде $d W(t) \rightarrow \xi(t) d t$, где величина $\xi(t)$ имеет $\delta$-функциональную корреляцию по времени, т. е. $\left\langle\xi(t) \xi\left(t^{\prime}\right)\right\rangle=$ $\delta\left(t-t^{\prime}\right)$, и вычислим средние в терминах величины $\xi$, что представляет собой в точности процедуру, применяемую при вычислениях с обычными правилами Фейнмана. Применяя эти правила, получим, что в появляющихся интегралах $\delta$-функции корреляций величин $\xi$ интегрируются таким образом, что один из пределов интегрирования совпадает с одним из аргументов $\delta$-функции. Можно явно увидеть, что в итеративных решениях типа древесных графов такие ситуации появляются исключительно благодаря присутствию временно́й функции ступеньки в пропагаторе. Эти неоднозначности разрешаются разными способами в определениях стохастического интеграла Ито и Стратоновича. Правила Ито в точности соответствуют выбору $\theta(0)=0$, а в правилах Стратоновича имеем $\theta(0)=1 / 2$.

Можно тем самым сформулировать следующие предписания при построении решения СДУ Стратоновича (1). Решение этого уравнения задается представлением в виде функционального интеграла либо с динамическим действием в форме Ито

$$
S^{\prime}[\varphi, \tilde{\varphi}, f]=-\frac{1}{2} f D^{-1} f+\tilde{\varphi}\left[-\partial_{t} \varphi-K \varphi+U(\varphi)+\frac{1}{2} B^{\prime}(\varphi) D B(\varphi)+f B(\varphi)\right],
$$


в котором $\Delta\left(0, \mathbf{x}-\mathbf{x}^{\prime}\right)=0$ и

$$
\begin{aligned}
\frac{1}{2} \tilde{\varphi} B^{\prime}(\varphi) D B(\varphi)=\frac{1}{2} & \int d t \int d \mathbf{x} \int d \mathbf{x}^{\prime} \tilde{\varphi}(t, \mathbf{x}) \times \\
& \times \int d u \frac{\delta B(\mathbf{x}, \varphi(u))}{\delta \varphi\left(t, \mathbf{x}^{\prime}\right)} D\left(\mathbf{x}-\mathbf{x}^{\prime}\right) B\left(\mathbf{x}^{\prime}, \varphi(t)\right)
\end{aligned}
$$

либо с динамическим действием в виде Стратоновича

$$
\begin{aligned}
S^{\prime \prime}[\varphi, \tilde{\varphi}, f]=- & \frac{1}{2}\left[U^{\prime}(\varphi)+\frac{1}{4} B^{\prime}(\varphi) D B^{\prime}(\varphi)+B^{\prime}(\varphi) f\right]- \\
& -\frac{1}{2} f D^{-1} f+\tilde{\varphi}\left[-\partial_{t} \varphi-K \varphi+U(\varphi)+f B(\varphi)\right],
\end{aligned}
$$

в котором $\Delta\left(0, \mathbf{x}-\mathbf{x}^{\prime}\right)=\delta\left(\mathbf{x}-\mathbf{x}^{\prime}\right) / 2$ и

$$
\frac{1}{2}\left[U^{\prime}(\varphi)+B^{\prime}(\varphi) f\right]=\frac{1}{2} \int d t \int d u \int d \mathbf{x}\left[\frac{\delta U(\mathbf{x}, \varphi(u))}{\delta \varphi(t, \mathbf{x})}+\frac{\delta B(\mathbf{x}, \varphi(u))}{\delta \varphi(t, \mathbf{x})} f(t, \mathbf{x})\right] .
$$

Из этих определений видно, что выбор действия Ито оказывается как более удобным с точки зрения практических вычислений в теории возмущений, так и более надежным при использовании в непертурбативных вычислениях функционального интеграла.

Следует отметить, что все моменты случайного поля $\varphi$, вычисленные с помощью этих двух динамических действий, в точности равны друг другу и представляют собой решение СДУ (1) в интерпретации Стратоновича, т. е. одно и то же решение может быть получено с применением двух различных динамических действий (20) и $(21)$.

\section{3. РЕШЕНИЕ УПРАВЛЯЮЩЕГО УРАВНЕНИЯ В ТЕОРИИ ПОЛЯ}

Рассмотрим реакцию коагуляции, ограниченную диффузией, $A+A \rightarrow A$ в подходе управляющего уравнения. Система управляющих уравнений для вероятностей $P\left(t,\left\{n_{i}\right\}\right)$ наблюдения $n_{i}$ частиц в узле с номером $i$ в момент времени $t$ имеет вид

$$
\begin{aligned}
\partial_{t} P( & \left.t,\left\{n_{i}\right\}\right)=\frac{D}{b^{2}} \sum_{\mathbf{e}}\left[\left(n_{i+\mathbf{e}}+1\right) P\left(t, n_{1}, \ldots, n_{i}-1, n_{i+\mathbf{e}}+1, \ldots\right)-n_{C} P\left(t,\left\{n_{i}\right\}\right)\right]+ \\
& +\frac{k}{V}\left[\left(n_{i}+2\right)\left(n_{i}+1\right) P\left(t, n_{1}, \ldots, n_{i}+2, \ldots\right)-\left(n_{i}+1\right) n_{i} P\left(t,\left\{n_{i}\right\}\right)\right],
\end{aligned}
$$

где сумма в правой части берется по ближайшим соседям узла $i$, в непрерывном пределе эта сумма переходит в диффузионный член.

Изменения в числе частиц удобно описать в терминах пространства Фока: применим операторы $\hat{a}_{i}, \hat{a}_{i}^{+}$и базисные векторы $\left|\left\{n_{i}\right\}\right\rangle$ :

$$
\hat{a}_{i}|0\rangle=0, \quad \hat{a}_{j}^{+}\left|\left\{n_{i}\right\}\right\rangle=\left|\left\{n_{i}+\delta_{i j}\right\}\right\rangle, \quad\left[\hat{a}_{i}, \hat{a}_{j}^{+}\right]=\delta_{i j} .
$$

Из управляющего уравнения получим для вектора состояния

$$
|\Phi\rangle=\sum_{n_{i}=0}^{\infty} P\left(t,\left\{n_{i}\right\}\right)\left|\left\{n_{i}\right\}\right\rangle
$$


следующее кинетическое уравнение:

$$
\frac{d|\Phi\rangle}{d t}=\hat{L}\left(\hat{a}^{+}, \hat{a}\right)|\Phi\rangle
$$

в котором оператор Лиувилля $\hat{L}$ задается системой управляющих уравнений в соответствии с правилами, установленными Дои в работах [8].

Представление функционального интеграла для математических ожиданий можно получить из кинетического уравнения в виде [11]

$$
\begin{aligned}
\left\langle Q\left(\left\{n_{i}\right\}\right)\right\rangle=\int & \int \prod_{i} \mathcal{D} a_{i}^{+} \mathcal{D} a_{i} e^{\left[S\left(a^{+}, a\right)+S_{\mathrm{in}}\left[a^{+}(0)\right]\right]} \times \\
& \times\left. Q_{N}\left[\left\{a_{i}^{+}(t)\right\}+1,\left\{a_{i}(t)\right\}\right]\right|_{a_{i}^{+}(t) \rightarrow 0},
\end{aligned}
$$

где динамическое действие задается оператором Лиувилля, взятым в нормальной форме, $S\left(a^{+}, a\right)=\int d t L_{N}\left(a^{+}+1, a\right)$, а второй член отвечает начальным условиям

$$
S_{\text {in }}\left[a^{+}(0)\right]=\ln \left\{\sum_{n_{i}=0}^{\infty} P\left(0,\left\{n_{i}\right\}\right) \prod_{i}\left[a_{i}^{+}(0)+1\right]^{n_{i}}\right\} .
$$

В непрерывном пределе операторы рождения и уничтожения заменяются полевыми операторами, а суммы по узлам решетки заменяются интегрированиями в соответствии со следующими правилами: $a_{i}^{+} \rightarrow \psi^{+}(\mathbf{x}), a_{i} / V \rightarrow \psi(\mathbf{x})$ и $\sum_{i} V \rightarrow \int d \mathbf{x}$, где объемный фактор $V$ имеет смысл элемента объема, предписанного узлу решетки.

Динамическое действие Дои для реакции коагуляции $A+A \rightarrow A$ имеет вид

$$
S\left[\psi^{+}, \psi\right]=\psi^{+}\left(-\partial_{t} \psi+D \nabla^{2} \psi-k \psi^{2}\right)-k\left(\psi^{+}\right)^{2} \psi^{2} .
$$

Сравним это динамическое действие с динамическим действием, возникающим в подходе уравнения Ланжевена. Явный вид действия Дои, не содержащий никаких указаний на вклад определителя, характерный для подхода СДУ, предполагает, что динамическое действие должно быть выбрано в форме Ито. СДУ Стратоновича для РОД $A+A \rightarrow A$ можно записать в виде

$$
\frac{\partial \varphi}{\partial t}=D \nabla^{2} \varphi-k \varphi^{2}+f \varphi
$$

с корреляционной функцией (здесь обозначения изменены из-за присутствия коэффициента диффузии)

$$
\left\langle f(t, \mathbf{x}) f\left(t^{\prime}, \mathbf{x}^{\prime}\right)\right\rangle=\delta\left(t-t^{\prime}\right) C\left(\mathbf{x}-\mathbf{x}^{\prime}\right) .
$$

Обращение в нуль члена с источником (24) при обращении в нуль $\varphi$ необходимо для того, чтобы пустое состояние оставалось абсорбирующим.

СДУ (24) и (25) приводят к динамическому действию в форме Ито:

$$
S[\varphi, \tilde{\varphi}]=\tilde{\varphi}\left(-\partial_{t} \varphi+D \nabla^{2} \varphi+\frac{1}{2} C(0) \varphi-k \varphi^{2}\right)+\frac{1}{2} \tilde{\varphi}^{2} C \tilde{\varphi}^{2} .
$$

Члены четвертого порядка в действиях (23) и (26) имеют различные знаки, так что их нельзя сделать равными с помощью выбора корреляционной функции $C$, 
которая должна быть положительной в случае реального шума. Шумовое поле $f$ можно формально выбрать в чисто мнимом виде, чтобы обеспечить правильный знак в действии (26), поскольку $f$ представляет собой вспомогательную величину. Тем не менее это представляется несколько искусственной конструкцией и указывает на то, что уравнение Ланжевена (24) описывает задачу, отличную от той, которая описывается управляющим уравнением (22).

\section{4. МОДЕЛЬ, ОГРАНИЧЕННАЯ ДИФФУЗИЕЙ, СОВРЕМЕННОГО БОЕСТОЛКНОВЕНИЯ}

Модели боестолкновений Ланчестера представляют собой модели реакций, описывающих абсолютные или относительные темпы боевых потерь. Эти модели были предложены Ланчестером [12] в 1914 г. и независимо Осиповым [13] в 1915 г.

Модель Ланчестера современного боестолкновения основана на идее концентрации направленного огня на отдельные вражеские единицы в каждый отдельный момент времени. Это означает, что скорость вражеских потерь не зависит от его военной силы - величины, описывающей эффективности различных армейских подразделений, и эта скорость пропорциональна силе противостоящей стороны. Дифференциальные уравнения, описывающие силы Красной Армии $\left(n_{\mathrm{r}}\right)$ и Голубой Армии $\left(n_{\mathrm{b}}\right)$ в модели Ланчестера имеют вид

$$
\frac{d n_{\mathrm{r}}}{d t}=-\alpha_{\mathrm{r}} n_{\mathrm{b}}, \quad \frac{d n_{\mathrm{b}}}{d t}=-\alpha_{\mathrm{b}} n_{\mathrm{r}}
$$

где $\alpha_{\mathrm{r}}$ и $\alpha_{\mathrm{b}}$ - коэффициенты вооружений. Уравнения Ланчестера (27) описывают динамику усредненных потерь. Реальное боестолкновение, однако, всегда содержит элементы случайности, приводящие к стохастическим процессам.

Управляющие уравнения для функций распределения вероятностей $P\left(t, n_{\mathrm{r}}, n_{\mathrm{b}}\right)$ стохастической модели современного боестолкновения имеют вид

$$
\begin{aligned}
\frac{d P\left(t, n_{\mathrm{r}}, n_{\mathrm{b}}\right)}{d t}= & \alpha_{\mathrm{r}} n_{\mathrm{b}}\left[P\left(t, n_{\mathrm{r}}+1, n_{\mathrm{b}}\right)-P\left(t, n_{\mathrm{r}}, n_{\mathrm{b}}\right)\right]+ \\
& +\alpha_{\mathrm{b}} n_{\mathrm{r}}\left[P\left(t, n_{\mathrm{r}}, n_{\mathrm{b}}+1\right)-P\left(t, n_{\mathrm{r}}, n_{\mathrm{b}}\right)\right], \quad n_{\mathrm{r}}, n_{\mathrm{b}} \geqslant 1,
\end{aligned}
$$

Для описания процесса уничтожения, протекающего со скоростью, не зависящей от числа заполнения $n$, необходимо ввести специальный оператор уничтожения $\hat{A}$ [14], [15]:

$$
\hat{A}|0\rangle=0, \quad \hat{A}|n\rangle=|n-1\rangle, \quad n \geqslant 1 .
$$

Специальный оператор, записанный в нормальной форме, имеет вид

$$
\hat{A}=\sum_{n=1}^{\infty} \frac{(-1)^{n-1}}{n !}\left(\hat{a}^{+}\right)^{n-1} \hat{a}^{n} .
$$


С применением этого представления динамическое действие Дои для модели боестолкновения Ланчестера, ограниченной диффузией, принимает вид

$$
\begin{aligned}
S\left(\psi_{\mathrm{r}}^{\dagger}, \psi_{\mathrm{r}}, \psi_{\mathrm{b}}^{\dagger}, \psi_{\mathrm{b}}\right)= & -\psi_{\mathrm{r}}^{\dagger} \partial_{t} \psi_{\mathrm{r}}+D_{\mathrm{r}} \psi_{\mathrm{r}}^{\dagger} \nabla^{2} \psi_{\mathrm{r}}-\psi_{\mathrm{b}}^{\dagger} \partial_{t} \psi_{\mathrm{b}}+D_{\mathrm{b}} \psi_{\mathrm{b}}^{\dagger} \nabla^{2} \psi_{\mathrm{b}}- \\
& -\alpha_{\mathrm{r}}\left(1+\psi_{\mathrm{b}}^{\dagger}\right) \psi_{\mathrm{b}} \psi_{\mathrm{r}}^{\dagger} \sum_{n=0}^{\infty} \frac{(-1)^{n}}{(n+1) !}\left(1+\psi_{\mathrm{r}}^{\dagger}\right)^{n} \psi_{\mathrm{r}}^{n+1}- \\
& -\alpha_{\mathrm{b}}\left(1+\psi_{\mathrm{r}}^{\dagger}\right) \psi_{\mathrm{r}} \psi_{\mathrm{b}}^{\dagger} \sum_{n=0}^{\infty} \frac{(-1)^{n}}{(n+1) !}\left(1+\psi_{\mathrm{b}}^{\dagger}\right)^{n} \psi_{\mathrm{b}}^{n+1}
\end{aligned}
$$

Сумма масштабных размерностей основного и сопряженного полей в действии (30) равна размерности пространства: $d_{\psi}+d_{\psi^{\dagger}}=d$. При взгляде на графы становится понятным, что $d_{\psi_{\mathrm{r}}}=d_{\psi_{\mathrm{b}}}=0$; тогда для логарифмической размерности модели современного боестолкновения получим, что $d_{\mathrm{c}}=2$. Подсчет степеней для графов показывает, что процедура перенормировки приводит к появлению новых УФ-расходящихся структур.

Заметим, однако, что с помощью явной однопетлевой перенормировки можно выделить подмножество членов, управляющих асимптотическим поведением модели на больших масштабах и приводящих к мультипликативно перенормируемой модели с конечным числом контролирующих параметров [14]. Члены мультипликативно перенормированного действия, которые остаются ненулевыми в нетривиальной фиксированной точке однопетлевой перенормировки, составляют эффективное действие

$$
\begin{aligned}
S_{\mathrm{eff}}= & -\psi_{\mathrm{r}}^{\dagger} \partial_{t} \psi_{\mathrm{r}}+D_{\mathrm{r}} \psi_{\mathrm{r}}^{\dagger} \nabla^{2} \psi_{\mathrm{r}}-\psi_{\mathrm{b}}^{\dagger} \partial_{t} \psi_{\mathrm{b}}+D_{\mathrm{b}} \psi_{\mathrm{b}}^{\dagger} \nabla^{2} \psi_{\mathrm{b}}- \\
& -\alpha_{\mathrm{rb}} \psi_{\mathrm{r}}^{\dagger} \psi_{\mathrm{r}} \psi_{\mathrm{b}}^{\dagger} \psi_{\mathrm{b}}-\psi_{\mathrm{b}} \psi_{\mathrm{r}}^{\dagger} \sum_{n=0}^{\infty} \frac{\alpha_{\mathrm{r} n} \psi_{\mathrm{r}}^{n+1}}{(n+1) !}-\psi_{\mathrm{r}} \psi_{\mathrm{b}}^{\dagger} \sum_{n=0}^{\infty} \frac{\alpha_{\mathrm{b} n} \psi_{\mathrm{b}}^{n+1}}{(n+1) !}
\end{aligned}
$$

Исследование графов показывает, что это действие является мультипликативно перенормируемым с константами перенормировки, зависящими только от константы связи $\alpha_{\mathrm{rb}}$. На уровне однопетлевого приближения это действие имеет фиксированную точку

$$
\alpha_{\mathrm{rb}}^{*}=2 \pi\left(D_{\mathrm{r}}+D_{\mathrm{b}}\right) \varepsilon
$$

которая оказывается ИК-стабильной при $\varepsilon=2-d>0$.

Эффективное действие (31) все еще содержит бесконечное число параметров, но если выбрать остающиеся ряды в исходном виде (30), то останутся свободными только три параметра $\alpha_{\mathrm{r}}, \alpha_{\mathrm{b}}$ и $\alpha_{\mathrm{rb}}$ в функционале взаимодействия (31) и коэффициенты диффузии $D_{\mathrm{r}}$ и $D_{\mathrm{b}}$ в свободной части действия; асимптотическое поведение вакуумных ожиданий полностью описывается этими параметрами.

Асимптотическое поведение среднего уровня силы можно получить из решения уравнения ренормализационной группы в виде

$$
\begin{array}{r}
n_{\mathrm{r}, \mathrm{b}}\left(t,\left\{\alpha_{\mathrm{r} n}^{R}\right\},\left\{\alpha_{\mathrm{b} n}^{R}\right\}, \alpha_{\mathrm{rb}}^{R}\right)=n_{\mathrm{r}, \mathrm{b}}\left(\tau,\left\{\bar{\alpha}_{\mathrm{r} n}\right\},\left\{\bar{\alpha}_{\mathrm{b} n}\right\}, \bar{\alpha}_{\mathrm{rb}}\right) \sim \\
\sim n_{\mathrm{r}, \mathrm{b}}\left(\tau,\left\{\left(\alpha_{\mathrm{r} n} \tau\right)(t / \tau)^{d / 2}\right\},\left\{\left(\alpha_{\mathrm{b} n} \tau\right)(t / \tau)^{d / 2}\right\}, \alpha_{\mathrm{rb}}^{*}\right)
\end{array}
$$


с инвариантными параметрами

$$
\begin{aligned}
2 t \frac{d}{d t} \bar{\alpha}_{\mathrm{rb}} & =\bar{\alpha}_{\mathrm{rb}}\left[\varepsilon+\gamma_{\mathrm{rb}}\left(\bar{\alpha}_{\mathrm{rb}}\right)\right] \\
2 t \frac{d}{d t} \bar{\alpha}_{\mathrm{r} n} & =\bar{\alpha}_{\mathrm{r} n}\left[2+\gamma_{\mathrm{rb}}\left(\bar{\alpha}_{\mathrm{rb}}\right)\right] \\
2 t \frac{d}{d t} \bar{\alpha}_{\mathrm{b} n} & =\bar{\alpha}_{\mathrm{b} n}\left[2+\gamma_{\mathrm{rb}}\left(\bar{\alpha}_{\mathrm{rb}}\right)\right] .
\end{aligned}
$$

Ввиду неограниченного роста параметров $\bar{\alpha}_{\mathrm{r} n}$ и $\bar{\alpha}_{\mathrm{b} n}$ с ростом времени, следует взять сумму их степеней роста, чтобы получить настоящее асимптотическое поведение. Эта задача выходит за рамки подхода ренормализационной группы, и она не была решена даже в более простом случае бинарной реакции $A+B \rightarrow \varnothing[16]$.

Ведущим приближением к решению задачи о среднем уровне силы могло бы быть решение стационарных уравнений для динамического действия (31). Однако в замкнутом виде не было получено ни одного решения этих уравнений. В настоящее время наилучшим асимптотическим результатом остается асимптотическое поведение, вычисленное на основе приближенных уравнений стационарности в критическом случае $\left(n_{\mathrm{r}}(\tau)=n_{\mathrm{b}}(\tau), \alpha_{\mathrm{r} n}=\alpha_{\mathrm{b} n}=\alpha\right.$ для всех $\left.n\right)$ соответственно для высоких и низких уровней силы:

$$
n_{\mathrm{r}}(t) \sim n_{\mathrm{r}}(\tau) e^{-\alpha \tau(t / \tau)^{d / 2}}, \quad n_{\mathrm{r}}(t) \sim \frac{1}{\alpha \tau(t / \tau)^{d / 2}} .
$$

Эти выражения получены в предположении, что решения в виде древесных графов для средних уровней силы правильно описывают зависимость от времени ведущих степеней, а петлевые поправки обусловливают поправки к амплитудам и к низшим степеням временно́й зависимости. Доказательство этой гипотезы на настоящий момент отсутствует, так что для обоснования выражений (34) необходимо принять ее в качестве предположения.

\section{5. ЗАКЛЮЧЕНИЕ}

В работе проанализированы неоднозначности, возникающие в решениях функционально-интегрального уравнения типа СДУ. Представление решения в виде функционального интеграла приводит к неоднозначности при выборе начального значения пропагатора, которая имеет место даже в случае детерминистского уравнения. Из математической несогласованности СДУ с белым шумом по времени следуют интерпретации СДУ в смыслах Ито и Стратоновича. Показано, что функциональноинтегральные решения обоих СДУ можно построить с помощью двух динамических действий, отличающихся выбором начального значения пропагатора и детерминистской части динамического действия. Разница в детерминистских частях двух динамических действий отвечает взаимосвязи между СДУ Ито и Стратоновича, которые приводят к одним и тем же решениям. Предложены аргументы в пользу того, что динамическое действие в форме Ито оказывается предпочтительным.

На основе реакции коагуляции $A+A \rightarrow A$ мы обсудили соотношение между полевыми теориями, возникающими из описаний стохастических задач с помощью уравнения Ланжевена и с помощью управляющего уравнения. 
Особенности стохастической полевой теории, используемой для описания процесса уничтожения со скоростью перехода, не зависящей от плотности реагента, обсуждены на примере модели боестолкновения Ланчестера, ограниченной диффузией.

\section{Список литературы}

[1] C. De Dominicis, J. Phys. Colloques, 37:C1 (1976), 247-254; H.-K. Janssen, Z. Phys. B, 23:4 (1976), 377-380.

[2] P. C. Martin, E. D. Siggia, H. A. Rose, Phys. Rev. A, 8:1 (1973), 423-437.

[3] G. Muñoz, W. S. Burgett, J. Stat. Phys., 56:1-2 (1989), 59-68; M. Chaichian, A. Demichev, Path Integrals in Physics, v. I: Stochastic Processes and Quantum Mechanics, IOP Publishing, Bristol, 2001.

[4] J. Zinn-Justin, Quantum Field Theory and Critical Phenomena, Internat. Ser. Monog. Phys., New York, Oxford Univ. Press, 1996.

[5] А.Н. Васильев, Квантовополевая ренормгруппа в теории критического поведения и стохастической динамике, Изд-во ПИЯФ, СПб., 1998.

[6] C. W. Gardiner, Handbook of Stochastic Methods for Physics, Chemistry and the Natural Sciences, Springer Series in Synergetics, 13, Springer, Berlin, 1997.

[7] N.G. van Kampen, Stochastic Processes in Physics and Chemistry, North-Holland, Amsterdam, 1984.

[8] M. Doi, J. Phys. A:, 9:9 (1976), 1465-1477; 1479-1495.

[9] L. Peliti, J. Phys. (Paris), 46:9 (1985), 1469-1484.

[10] P. C. Hohenberg, B. I. Halperin, Rev. Mod. Phys., 49:3 (1977), 435-479.

[11] M. Hnatich, J. Honkonen, Phys. Rev. E, 61:4 (2000), 3904-3911.

[12] F. W. Lanchester, Engineering, 98 (1914), 422-423.

[13] М. Осипов, Военный сборник, 6 (1915), 59-74; 7 (1915), 25-36; 8 (1915), 31-40; 9 (1915), 25-37; 10 (1915), 93-96.

[14] J. Honkonen, Warfare in Fock Space, National Defence College, Helsinki, 2003.

[15] J. Honkonen, Phys. Part. Nucl. Lett., 5:3 (2008), 196-200.

[16] B. P. Lee, J. Cardy, J. Stat. Phys., 80:5-6 (1995), 971-1007; 87:3-4 (1997), 951-954. 